\title{
Setting Time as Purely Imaginary in Regime of Space-Time before Causal Barrier and Planckian Space-Time
}

\author{
Andrew Walcott Beckwith \\ Physics Department, College of Physics, Chongqing University, Huxi Campus, Chongqing, China \\ Email: Rwill9955b@gmail.com, abeckwith@uh.edu
}

How to cite this paper: Beckwith, A.W. (2018) Setting Time as Purely Imaginary in Regime of Space-Time before Causal Barrier and Planckian Space-Time. Journal of High Energy Physics, Gravitation and Cosmology, 4, 92-95.

https://doi.org/10.4236/jhepgc.2018.41009

Received: December 17, 2017

Accepted: January 21, 2018

Published: January 24, 2018

Copyright $\odot 2018$ by author and Scientific Research Publishing Inc. This work is licensed under the Creative Commons Attribution International License (CC BY 4.0).

http://creativecommons.org/licenses/by/4.0/

\begin{abstract}
We start where we use an inflaton value due to use of a scale factor $a \sim a_{\min } t^{\gamma}$. In doing so, using the approximations brought up in the case of an early universe generation of a magnetic field, we come up with the stunning datum of a Pre Planckian space-time with purely imaginary time. The consequences of such are brought up in our document. We use what is brought up in a prior article as to Pre Planckian to Planckian generation of Magnetic fields to make our point. Here, we assume, in the Pre Planckian space-time, a singular injection of space-time, which we argue, if there is no spatial variation of the Inflaton field, so used, and we just have ONE Pre Planckian time instant that this will be imaginary valued time, hence leading to pure Tunneling behavior. Whatever we are assuming, using a Penrose cosmology to initiate CCC universe recycling, in terms of energy, is transferred to Planckian space-time.
\end{abstract}

\section{Keywords}

Inflaton Physics, Causal Structure

\section{Examining the Pre Planckian Inflaton, and Its Relationship to as Hamiltonian}

We begin with using the inflaton as given by [1] with the result that we will be looking at the Pre Planckian potential and inflaton system as looking like

$$
\begin{aligned}
& a \approx a_{\min } t^{\gamma} \\
& \Leftrightarrow \phi \approx \sqrt{\frac{\gamma}{4 \pi G}} \cdot \ln \left\{\sqrt{\frac{8 \pi G V_{0}}{\gamma \cdot(3 \gamma-1)}} \cdot t\right\} \\
& \Leftrightarrow V \approx V_{0} \cdot \exp \left\{-\sqrt{\frac{16 \pi G}{\gamma}} \cdot \phi(t)\right\} .
\end{aligned}
$$

Using the approximation of a log expansion of the inflaton, this leads to, for 
approximate small space-time

$$
\phi \approx \sqrt{\frac{\gamma}{4 \pi G}} \cdot\left\{1-1 / \sqrt{\frac{8 \pi G V_{0}}{\gamma \cdot(3 \gamma-1)}} \cdot t\right\} .
$$

We next then, as discussed in [1] make the assumption of a modified CCC Penrose style cyclic conformal cosmology recycling of Space-time into the interior bubble where Equation (1) holds, and we use the following Hamiltonian system [2]

$$
H(\inf )=\int \mathrm{d}^{(n-1)} x \cdot\left[\frac{\dot{\phi}^{2}+(\nabla \phi)^{2}+m^{2} \phi^{2}}{2}\right]
$$

The approximation we use is that there is, in the Pre Planckian space-time no spatial variation of the Inflaton, and in addition, that we just have imposed, due to our modified CCC Penrose style cyclic conformal cosmology recycling of Space-time into the interior bubble, just ONE instant of time, i.e. then what we work with is, with approximations.

$$
H(\text { inf })=\left(\int \mathrm{d}^{(n-1)} x\right) \cdot\left[\frac{\dot{\phi}^{2}+m^{2} \phi^{2}}{2}\right] .
$$

Using the assumption that if there is just ONE time instant, as similar to the Hamiltonian being TIME independent, we write the above as $H=E$ (energy), [3] We then, using Equation (4), for $H=E$, Equation (2) for an Inflaton, and $\left(\int \mathrm{d}^{(n-1)} x\right)=V(n-1 \mathrm{dim})$, reduce our time Equation to look like the following, i.e. we will define $m$, after we set the square of Time in the Pre Planckian regime

$$
\begin{aligned}
& \left(t^{2}\right)^{2}-\left(1+m^{2}\left(t^{2}\right)\right) \cdot\left(\frac{\gamma \cdot(3 \gamma-1) \cdot V(n-1 \mathrm{dim})}{16 \pi^{2} G^{2} V_{0} E}\right)=0 \\
& \& A=\frac{\gamma \cdot(3 \gamma-1) V(n-1 \mathrm{dim})}{16 \pi^{2} G^{2} V_{0} E} \\
& \Rightarrow\left(t^{2}\right)=\frac{m^{2} A}{2} \cdot\left(1 \pm \sqrt{1+\frac{1}{m^{4} A}}\right) \\
& \&\left(t^{2}\right) \sim-\frac{1}{4 m^{2}} .
\end{aligned}
$$

This last line of Equation (5) is for the Pre Planckian regime of space-time, and in it we will by [1] make the following identification.

$$
m \sim N(\text { Entropy-count }) \cdot m_{g} \text {. }
$$

We will in our conclusion elaborate as to what this may be leading to.

\section{Imaginary Time? In Pre Planckian Space?}

It is a truism, that in Field theory, imaginary time inverts potentials. Moreover it is also a way to discuss barrier penetration problems, i.e. this is done time and time again in Quantum Mechanics [4], and in field theory [5].

Without further ado, we state that this imaginary time designation, as forced, 
for ONE instant of space-time is equivalent to reducing the problem, in terms of Pre Planckian space-time for ONE given instant of time, put into our $H=E$ identification, is the same as having a barrier penetration forced from Pre Planckian to Planckian Space time.

\section{Conclusions. The Transformation from Imaginary Time, in Pre Planckian to Planckian Space Time Real Time, Presages Why We Have a Causal Barrier, and Is Profoundly Important}

What we need to do, in our work, is to work in terms of generalizing what we took from [6] as far as our modification of Penrose, that we are assuming a Pre Planckian space-time to be the recipient of energy from Multiverse contributions, i.e. to finalize our approximations used in this note, we need to delineate further, what this is saying, as it also relates to the issues brought up in [1] as to a Pre-Planckian to Planckian Universe generated magnetic field.

However, if the characterization for time varies this radically between the Pre Planckian to Planckian Universe, and this is upheld, then we have more basis as to forming appropriate conditions as to early universe magnetic fields, and may afterwards answer the questions raided in [6] [7] and [8] as well as [1]. Also the issues of [9] [10] [11] can be reviewed again, i.e. for [9] the issue if we can or cannot devise (experimentally useful?) filters can be reviewed again, due to what was said in [1].

In addition, we should not have hypothesis which flatly contradicts what was seen experimentally in [10] [11] either.

\section{Acknowledgements}

This work is supported in part by National Nature Science Foundation of China grant No. 1137527.

\section{References}

[1] Beckwith, A.W. (2017) How to Determine a Jump in Energy Prior to a Causal Barrier, with an Attendant Current, for an Effective Initial Magnetic Field in the Pre Planckian to Planckian Space-Time. http://vixra.org/abs/1707.0250

[2] Carrroll, S. (2003) Spacetime and Geometry, an Introduction to General Relativity. Addison and Westley, Menlo Park.

[3] Landau, D. and Liftshitz, E. (2005) Mechanics. 3rd Edition, Elsevier Press, Philadelphia.

[4] Landau, D. and Liftshitz, E. (1981) Quantum Mechanics, Non Relativistic. 3rd Edition, Pergamum Press, Oxford.

[5] Peskins, M.E. and Schroeder, D. (1995) An Introduction to Quantum Field Theory. Perseus Books, Menlo Park.

[6] Beckwith, A.W. (2014) Analyzing Black Hole Super-Radiance Emission of Particles/Energy from a Black Hole as a Gedankenexperiment to Get Bounds on the Mass of a Graviton. Advances in High Energy Physics, 2014, Article ID: 230713. 
https://doi.org/10.1155/2014/230713

[7] Corda, C. and Cuesta, H. (2010) Removing Black Hole singularities with Non Linear Electrodynamics. Modern Physics A, 25, 2423-2429

[8] Corda, C. (2009) Interferometric Detection of Gravitational Waves: The Definitive Test for General Relativity. International Journal of Modern Physics D, 18, 2275-2282. https://doi.org/10.1142/S0218271809015904

[9] Hughes, S. (1998) Gravitational-Wave Astronomy Aspects of the Theory of Binary Sources and Interferometric Detectors. PhD Dissertation, California Institute of Technology, Pasadena. http://web.mit.edu/sahughes/www/thesis.pdf

[10] Abbott, B.P., et al. (2016) Observation of Gravitational Waves from a Binary Black Hole Merger. Physical Review Letters, 116, Article ID: 061102.

https://physics.aps.org/featured-article-pdf/10.1103/PhysRevLett.116.061102 https://doi.org/10.1103/PhysRevLett.116.061102

[11] Abbott, B.P., et al. (2016) GW151226: Observation of Gravitational Waves from a 22-Solar-Mass Binary Black Hole Coalescence. Physical Review Letters, 116, Article ID: 241103. https://doi.org/10.1103/PhysRevLett.116.241103 